\title{
A strategy to determine off-axis dosimetric leaf gap using OSLD and EPID
}

\author{
Janahiraman Divyalakshmi ${ }^{1}, K_{\text {Kohamathu Rafic }}^{1,2}$, Ebenezer Suman Babu, Timothy Peace Balasingh ${ }^{1}$, \\ Amalan Sebastin ${ }^{1}$, Christopher J. Sujith ${ }^{1}$, L Jose Solomon Raj ${ }^{1}$ \\ ${ }^{1}$ Department of Radiation Oncology, Christian Medical College, Vellore, Tamil Nadu, India \\ ${ }^{2}$ Regional Cancer Centre, Govt. Rajaji Hospital, Madurai, Tamil Nadu, India
}

\begin{abstract}
Background: The aim of the study was to investigate the dosimetric feasibility of using optically stimulated luminescence dosimeters (OSLD) and an electronic portal imaging device (EPID) for central axis (CAX) and off-axis (OAX) dosimetric leaf gap (DLG) measurement.

Materials and methods: The Clinac 2100C/D linear accelerator equipped with Millennium-120 multileaf collimator (MLC) and EPID was utilized for this study. The DLG values at CAX and $\pm 1 \mathrm{~cm}$ OAX (1 cm superior and inferior to the CAX position, respectively along the plane perpendicular to MLC motion) were measured using OSLD (DLG $\mathrm{osLD}_{\text {) }}$ and validated using ionization chamber dosimetry ( $D L G_{\mathrm{ICD}}$ ). The two-dimensional DLG map (2D DLG $\mathrm{EPPO}_{\mathrm{O}}$ ) was derived from the portal images of the DLG plan using a custom-developed software application that incorporated sliding aperture-specific correction factors.

Results: DLG OSLD and DLG $G_{C D}$ though measured with diverse setup in different media, showed similar variation both at CAX

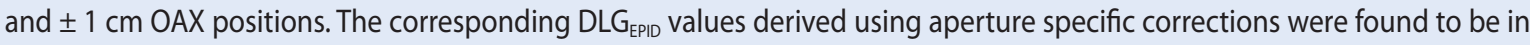
agreement with $D L G_{\text {osLD }}$ and $D L G_{\text {ICD. }}$. The $2 D D^{2} G_{\text {EPIO }}$ map provides insight into the varying patterns of the DLG with respect to each leaf pair at any position across the exposed field.

Conclusions: Commensurate results of DLG ${ }_{\text {osLD }}$ with DLG $\mathrm{GCD}_{\mathrm{CD}}$ values have proven the efficacy of OSLD as an appropriate dosimeter for DLG measurement. The $2 \mathrm{D} \mathrm{DLG}$ EPD map opens a potential pathway to accurately model the rounded-leaf end transmission with discrete leaf-specific DLG values for commissioning of a modern treatment planning system.

Key words: dosimetric leaf gap; rounded leaf-end transmission; electronic portal imaging device; optically stimulated luminescence dosimeter
\end{abstract}

Rep Pract Oncol Radiother 2021;26(6):1019-1028

\section{Introduction}

Radiation therapy has been transformed by the concept of conformal therapy with the establishment of the multileaf collimator (MLC) leading to improved targeting of the tumor [1]. The dynamic MLC system has progressed to facilitate complex dose delivery techniques, such as intensity modu- lated radiotherapy (IMRT) [2] and, more recently, volumetric modulated arc therapy (VMAT), where the movements of the MLC and gantry are simultaneous [3]. The accuracy of these sophisticated treatment planning and delivery techniques is directly dependent on accurate geometric modelling and incorporation of dosimetric characteristics of the dynamic MLCs in the dose calculation algorithm.

Address for correspondence: Timothy Peace Balasingh, PhD, Lecturer in Radiation Physics, tel: +91-9843982384;

e-mail: timothy@cmcvellore.ac

This article is available in open access under Creative Common Attribution-Non-Commercial-No Derivatives 4.0 International (CC BY-NC-ND 4.0) license, allowing to download articles and share them with others as long as they credit the authors and the publisher, but without permission to change them in any way or use them commercially 
Although a constant penumbra width is attained by the rounded end-leaf design $[4,5]$, the radiation transmission through the abutting leaves is an important parameter to be considered in the radiation beam transport, since the Eclipse ${ }^{\mathrm{TM}}$ treatment planning system (TPS) models the leaf-end as "square" in the optimization algorithm.

The dosimetric leaf gap (DLG), specific to the round-end leaf, is the small increase in field size at the isocentre perpendicular to the beam axis [6] and is incorporated in the TPS to convert the optimal fluence (computed for squared leaf-ends) into the actual fluence by shifting the leaf tips (in terms of fluence) of each MLC-bank by half the DLG value [7]. Hence, for computing the accurate dose distribution with Eclipse ${ }^{\mathrm{TM}}$ TPS, it is vital to determine the DLG value for dynamic MLC systems to model the physical difference between the abutting squared-end and rounded-end leaf designs in terms of radiation transmission [8]. Several studies and methods have been published to measure the DLG using various detectors, such as the ionization chamber, electronic portal imaging device (EPID), diode array, film and diamond detector, among which ionization chamber dosimetry (ICD) is considered to be the "gold standard" method [6, 9-15]. There were, however, three practical problems encountered in ICD viz., (i) the requirement of an elaborate dosimetric set-up (ii) the volume-averaging effect and (iii) the lack of electronic equilibrium when used for sweeping dynamic fields with small widths [16-18].To overcome the limitations imposed by conventional ICD for DLG measurement, EPID dosimetry, with its simplified approach and high-resolution detector matrix [19], was reported previously, however, leaving a question of the low-dose under-response of the EPID, especially for the sweeping dynamic fields that lead to an under-estimation of the DLG value $[9,12]$. Recent publications have emphasized the optically stimulated luminescence dosimeter (OSLD) as a highly sensitive dosimeter for low dose range measurement [20-23] and for small fields [24], invoking its use as a novel alternative for measuring the DLG with a simple dosimetric setup and calibration.

A current limitation of commercially available TPS is the global modelling of the abutting leaf-end transmission with a single DLG value measured at the central axis (CAX) $[6,25,26]$. It does not take into account the off-axis (OAX) variation in the DLG value. Therefore, this study investigates the potential use of OSLD and an EPID for OAX DLG measurement. Also, a strategy to overcome the low-dose under-response of the EPID using appropriate correction factors was explored and a two-dimensional (2D) EPID-based DLG map was computed to accurately depict the adjacent leaf pair-specific DLG and its OAX variation across the field.

\section{Materials and methods}

All treatment plans were computed using the Eclipse $^{\mathrm{TM}}$ TPS (v10.0, Varian Medical Systems, Palo Alto, CA) and delivered with a Clinac 2100C/D linear accelerator (Varian Medical Systems, Palo Alto, CA) that is equipped with the Millennium ${ }^{\mathrm{TM}}$ 120-leaf tertiary collimator having single-focused MLCs with rounded leaf-ends.

To determine the DLG, a ten-field "DLG plan" consisting of three static fields viz., a reference open field and two completely blocked MLC fields (to determine the radiation transmission through MLC bank), in addition to seven sweeping dynamic fields of varying gap widths ranging from 2 to 20 $\mathrm{mm}[9,27]$ were computed. Each field consisted of a $10 \times 10 \mathrm{~cm}^{2}$ field aperture corresponding to the central 20 leaf pairs (each of width $5 \mathrm{~mm}$ at isocentre). All the ten fields were irradiated using the 6 MV photon beam of the linear accelerator equipped with an amorphous silicon EPID having an active area of $40 \times 30 \mathrm{~cm}^{2}(1024 \times 768$ detector matrix $)$ and corresponding to a detector resolution of 0.39 $\mathrm{mm}$. Since the EPID was calibrated at a source to detector distance (SDD) of $105 \mathrm{~cm}$, all the DLG measurements were performed at the same SDD.

Due to the low-dose under-response of the EPID, especially for the completely blocked MLC fields and the sweeping dynamic fields with small widths, the DLG plan was computed to deliver $500 \mathrm{MU}$ per field and the DLG was determined for the "zero millimetre" field width using the linear extrapolation method [27]. The DLG was obtained from the graph in which the output factor (OF) corrected for MLC transmission (MLCT) is plotted against its corresponding sweeping gap width $(\mathrm{w})$ using a combined formula from equations described in literature $[9,12]$.

$$
O F_{w, i, j}=\frac{D_{w, i, j}-D_{M L C T, i, j}\left(1-\frac{w}{L}\right)}{D_{r e f, i, j}}
$$




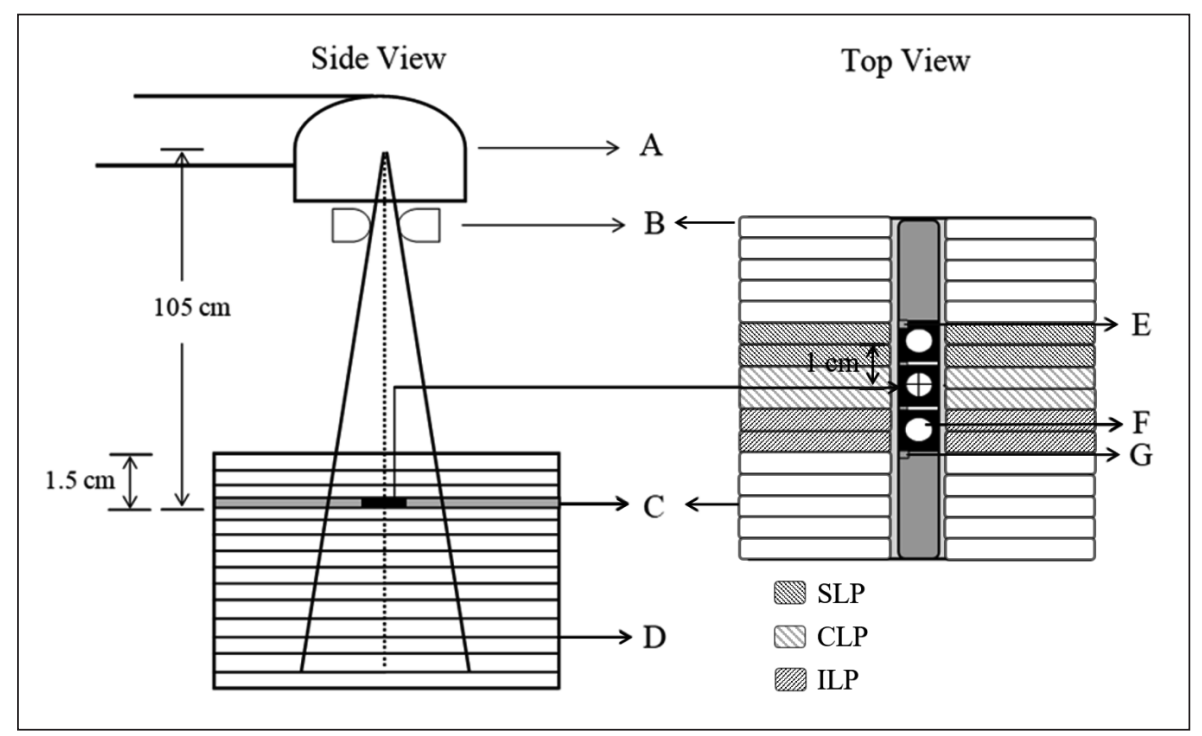

Figure 1. Schematic of dosimetric leaf gap (DLG) measurement using optically stimulated luminescence dosimeters (OSLD). A - gantry; B - MLC; C - 1D OSLD array; D - $10 \mathrm{~cm}$ Perspex Backscatter; E and G - narrow groove and tongue joint in OSLD; F - active OSLD phosphor; SLP — superior leaf pair; CLP — central leaf pair; ILP — inferior leaf pair

where,

- $\mathrm{OF}_{\mathrm{w}, \mathrm{i}, \mathrm{j}}$ is the output factor for a sweeping gap width " $w$ " corrected for MLCT at the $(i, j)^{\text {th }}$ position,

- $\mathrm{D}_{\mathrm{w}, \mathrm{i}, \mathrm{j}}$ is the dose measured for each sliding gap width,

- $\mathrm{D}_{\mathrm{MLCT}, \mathrm{i}, \mathrm{j}}$ is the mean dose measured with two completely closed MLC-bank fields,

- $\mathrm{D}_{\text {ref }, \mathrm{j}, \mathrm{j}}$ is the dose measured for reference field and

- $\mathrm{L}$ is the width of reference field

In the case of the 1D array measurements using OSLD as illustrated in Figure 1, " $\mathrm{j}$ " is taken as 1 while $i=1,2,3$ represents the three positions along the inplane direction as mentioned below (Fig.1). For EPID measurements, $(i, j)$ represents a pixel in the portal image matrix $(1024 \times 768$ pixels $)$.

In general, the DLG measured at isocentre, i.e. covering only the central leaf pair is used for modelling the entire leaf bank during the beam data configuration as part of the TPS commissioning process. However, in the first part of the present study, the DLG values corresponding to the six central leaf pairs were measured using OSLD, ICD and EPID (DLG OSLD $_{\text {, DLG }}$ and $\left.\mathrm{DLG}_{\mathrm{EPID}}\right)$. Three measurements viz., at CAX and $\pm 1 \mathrm{~cm}$ OAX ( $1 \mathrm{~cm}$ superior and inferior to the CAX position, respectively, along the plane perpendicular to MLC motion), were performed with each measurement covering two $5 \mathrm{~mm}$-adjacent leaf pairs (Fig. 1).
The DLG OSLD $_{\text {and }}$ DLG ${ }_{\text {ICD }}$ were calculated using two types of normalizations viz., the standard (or global) and the local normalization and compared. While the former method uses the dose measured at the CAX with reference open field for dose normalization, the latter uses the dose measured at the respective detector positions for the same.

\section{DLG with ICD (DLG $\left.{ }_{I C D}\right)$}

The conventional $D_{L G_{I C D}}$ measurements were performed in the same three positions (at CAX and $\pm 1 \mathrm{~cm} \mathrm{OAX)}$ as DLG OSLD with a $0.125 \mathrm{cc}$ Semiflex ionization chamber (PTW, Freiburg, Germany) "in-water" using the MP3-S therapy beam analyser (PTW, Freiburg, Germany). These were used to validate the DLG results from OSLD measurements $\left(D_{L} G_{\text {OSLD }}\right)$ as well as derive correction factors for accurate determination of DLG using the EPID (DLG $\left.\mathrm{DPII}_{\mathrm{EI}}\right)$.

\section{DLG with OSLD (DLG OSLD)}

In this study, the nanoDot ${ }^{\mathrm{sx}}$ OSLD (Landauer, Inc., Glenwood, IL), made of aluminium oxide doped with carbon $\left(\mathrm{Al}_{2} \mathrm{O}_{3}: \mathrm{C}\right)$, was used to measure the DLG. Each dosimeter consists of a $0.15 \mathrm{~mm}$ thick OSLD phosphor disc of diameter $5 \mathrm{~mm}$, sandwiched between two protective layers of polyester and encased in a light-tight plastic holder made of $0.5 \mathrm{~mm}$ thick acrylonitrile butadiene styrene. The $\mathrm{DLG}_{\text {OSLD }}$ measurements were carried out af- 
ter calibrating the OSLDs for doses ranging from 5 to $400 \mathrm{cGy}$ using the $6 \mathrm{MV}$ photon beam with a nominal dose rate of $400 \mathrm{MU} / \mathrm{min}$ for a reference field size of $10 \times 10 \mathrm{~cm}^{2}$ at a_source to surface distance (SSD) of $100 \mathrm{~cm}$. Three OSLDs were placed in a custom-made one-dimensional (1D) array (as shown in Fig. 1), permitting the centre-to-centre distance to be $1 \mathrm{~cm}$ at fixed SDD with $1.5 \mathrm{~cm}$ build up and $10 \mathrm{~cm}$ backscatter made of water-equivalent polymethyl methacrylate (PMMA) slabs to mimic the CAX and OAX DLG ICD $_{\text {measurements. }}$

\section{DLG with EPID (DLG EPID $_{\text {) }}$}

The $\mathrm{DLG}_{\mathrm{EPID}}$ values were derived from the ten portal images that were acquired through the DLG plan using the EPID. The mean dose from each portal image (in calibrated units, $\mathrm{CU}$ ) was acquired using the Portal Dosimetry software (v10.0, Varian Medical Systems, Palo Alto), within the specific ROI viz., $14 \times 16$ pixels, corresponding to the active area of the Semiflex chamber $\left(5.5 \times 6.5 \mathrm{~mm}^{2}\right)$ at the CAX and $\pm 1 \mathrm{~cm}$ OAX positions. Furthermore, to compare the DLG OSLD $_{\text {with }}$ DLG $_{\text {EPID }}$, the mean dose within an ROI of $13 \times 13$ pixels, corresponding to the OSLD phosphor of diameter $5 \mathrm{~mm}$, was also measured. In both measurements, the DLG values were obtained from the graphs plotted between the "w" and "OF" calculated as per equation (1). Hereafter, the DLG values derived from the ROIs corresponding to the active area of ICD and OSLD will be referred to as $\mathrm{DLG}_{\mathrm{EPID}, \mathrm{ICD}}$ and $\mathrm{DLG}_{\mathrm{EPID}, \mathrm{OSLD}}$, respectively.

\section{Correction factors for DLG}

In order to account for the low-dose under-response of the EPID, the relative dose-response of the EPID $\left(\mathrm{R}_{\mathrm{EPID}}, \mathrm{w}\right)$ was corrected to that of corresponding ICD $\left(\mathrm{R}_{\mathrm{ICD}}, \mathrm{W}\right)$ for the seven sweeping fields with varying gap widths. Due to the variation in dose response of EPID with respect to ICD for each sweeping gap width $(\mathrm{w})$, sliding aperture-specific correction factors $\left(\delta_{\mathrm{EPRD}, \mathrm{w}}\right)$ were incorporated into equation (1). The following equation (2), showing the OF corrected for under-response of EPID $\left(\mathrm{COF}_{\mathrm{w}, \mathrm{i}, \mathrm{j}}\right)$, was used for the accurate determination of DLG $\mathrm{EPID}_{\text {. }}$

$$
\begin{gathered}
c O F_{w, i, j}=\frac{\delta_{e p i d, w} D_{w, i, j}-D_{M L C T, i, j}\left(1-\frac{w}{L}\right)}{D_{r e f, i, j}} \\
\delta_{e p i d, w}=\frac{R_{I C D, w}}{R_{E P I D, w}}
\end{gathered}
$$

where, $R_{I C D, w}$ and $R_{E P I D, w}$ are the relative dose responses of ICD and EPID, for each sliding gap width.

\section{D DLG EPID $_{\text {map }}$}

To visualize the DLG variations across the field corresponding to the central 20-leaf pairs, a DICOM-handling script was coded in MATLAB (R2015a, Mathworks, USA). This software script converts the portal images obtained with the DLG plan into a 2D DLG area map in a four-step process as shown in Figure 2. At first, the image pixels of all the ten portal images are converted into dose pixels as per the formula given below.

$$
D_{i, j}=\frac{I_{i, j}-C}{m}
$$

where, $D_{i, j}$ and $I_{i, j}$ are the dose and pixel values at the position $(i, j)$, respectively, while " $C$ " and " $m$ " are the intercept and slope derived from the DICOM header of each portal image file.

Following this, the normalized OF (local normalization method) with corrections for both MLCT and $\delta_{\mathrm{EP} P, w}$, was generated for all the sweeping fields as per equation 2 . Next, the planar dose images of the sweeping fields were stacked into a 7-layered $3 \mathrm{D}$ array of dimension $1024 \times 768 \times 7$ pixels and permutated to a $768 \times 7 \times 1024$ matrix to facilitate regression analysis. Finally, an element-wise column operation was performed to derive the DLG value for each pixel through the regression value, slope and the intercept, and a 2D planar DLG distribution was generated.

\section{Results}

$$
\mathrm{DLG}_{\mathrm{ICD}}, \mathrm{DLG}_{\mathrm{OSLD}} \text { and DLG } \mathrm{EPID}_{\mathrm{E}}
$$

The DLG values measured with ICD and OSLD at CAX and OAX are given in Table 1. It was found that the DLG $\mathrm{ICD}_{\mathrm{ICD}}$ and $\mathrm{DLG}_{\mathrm{OSLD}}$ at CAX were similar $(\leq 1 \%)$ to that measured at the inferior OAX position while the same at the superior OAX position differed by $\geq 20 \%$. It was noticed that the DLG $\mathrm{OSLD}_{\mathrm{D}}$ values were comparable to $D_{L} G_{I C D}$ with a deviation of $-1.5 \%, 3.8 \%$ and $2.8 \%$ for the superior, central and inferior positions, respectively, when calculated using the global normalization method and a deviation of $-3.1 \%, 3.8 \%$ and $2.9 \%$ for the respective positions when calculated using the local normalization method. The DLG $\mathrm{EPID}_{\text {, ICD }}$ and $\mathrm{DLG}_{\mathrm{EPID} \text {, OsLD }}$ 


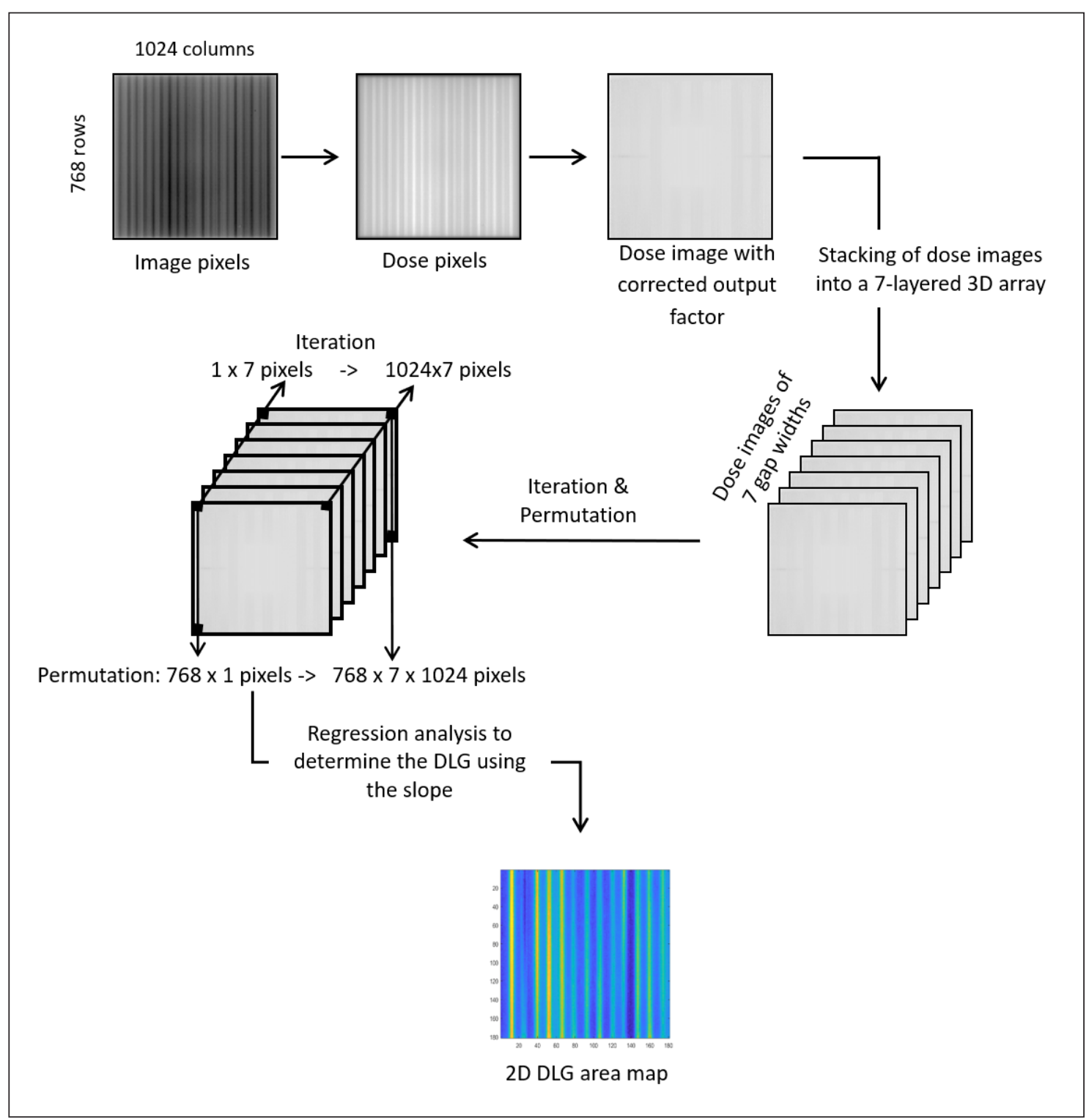

Figure 2. A workflow algorithm illustrating the generation of 2D DLG map from the portal images of the EPID

Table 1. Dosimetric leaf gap (DLG) measured at three positions using ionization chamber dosimetry (ICD) and optically stimulated luminescence dosimeters (OSLD) using two normalization methods

\begin{tabular}{|l|c|c|c|c|cc|}
\multirow{2}{*}{ Parameter } & \multicolumn{3}{c}{ Global normalization } \\
\cline { 2 - 7 } & Superior & Centre & Inferior & Superior & Centre & Inferior \\
\hline $\mathrm{DLG}_{\mathrm{ICD}}[\mathrm{mm}]$ & $1.27 \pm 0.02$ & $1.04 \pm 0.02$ & $1.05 \pm 0.01$ & $1.28 \pm 0.02$ & $1.04 \pm 0.02$ & $1.05 \pm 0.01$ \\
\hline $\mathrm{DLG}_{\text {OSLD }}[\mathrm{mm}]$ & $1.25 \pm 0.07$ & $1.08 \pm 0.03$ & $1.08 \pm 0.09$ & $1.24 \pm 0.04$ & $1.08 \pm 0.04$ & $1.08 \pm 0.06$ \\
\hline
\end{tabular}

(shown within the parentheses in Tab. 2), calculated for each detector position from the portal images using the ROI method, were found to vary from the reference $\mathrm{DLG}_{\mathrm{ICD}}$ by -13.5 to $-17.2 \%$ and -12.1 to $-18.5 \%$, respectively.

\section{Aperture-specific correction for DLG $G_{\text {EPID }}$}

The relative dose responses of the EPID $\left(\mathrm{R}_{\mathrm{EPID}, \mathrm{w}}\right)$ and the ICD $\left(\mathrm{R}_{\mathrm{ICD}, \mathrm{w}}\right)$ were found to vary from $4.6 \%$ to $0.6 \%$ as the sliding gap width increased from $2 \mathrm{~mm}$ to $20 \mathrm{~mm}$, respectively, as illustrated in 
Table 2. DLG EPID measured for ROI corresponding to ionization chamber dosimetry (ICD) and optically stimulated luminescence dosimeters (OSLD)

\begin{tabular}{|c|c|c|c|}
\hline Parameter & Superior & Centre & Inferior \\
\hline $\mathrm{DLG}_{\mathrm{EPID}, \mathrm{ICD}}[\mathrm{mm}]$ & $1.28 \pm 0.02(1.06 \pm 0.02)$ & $1.05 \pm 0.02(0.90 \pm 0.03)$ & $1.04 \pm 0.02(0.87 \pm 0.03)$ \\
\hline $\mathrm{DLG}_{\mathrm{EPID}, \mathrm{OSLD}}[\mathrm{mm}]$ & $1.28 \pm 0.03(1.09 \pm 0.02)$ & $1.05 \pm 0.02(0.91 \pm 0.02)$ & $1.04 \pm 0.01(0.88 \pm 0.01)$ \\
\hline
\end{tabular}

Uncorrected DLG $\mathrm{EPID}_{\mathrm{D}}$ values are given within parenthesis

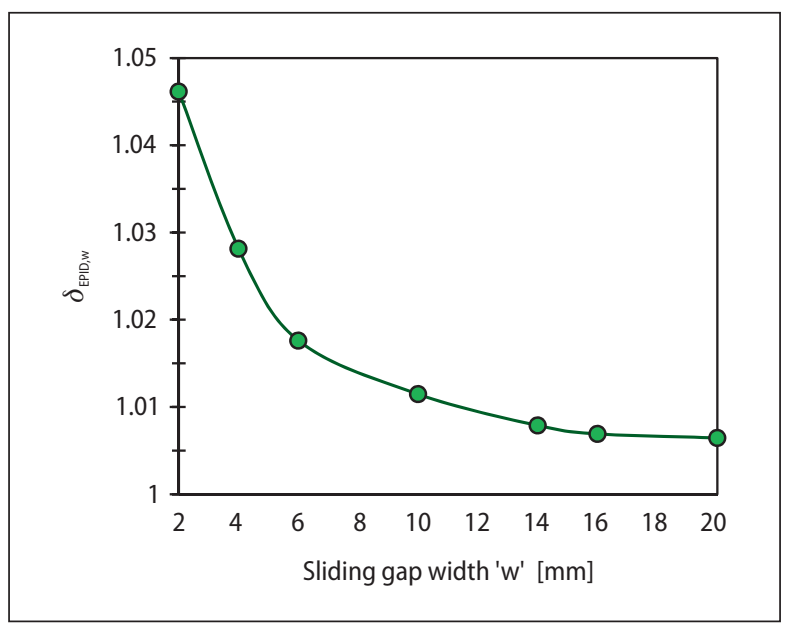

Figure 3. Sliding aperture-specific EPID response correction

Figure 3, and the corresponding fifth order polynomial fit is given in equation 5 .

$$
\begin{aligned}
y= & -3 E-08 x^{5}+3 E-06 x^{4}-0.0001 x^{3}+ \\
& +0.0024 x^{2}-0.0203 x+1.0781
\end{aligned}
$$

Therefore, for each detector position, the $\mathrm{DLG}_{\mathrm{EPID}, \mathrm{ICD}}$ and DLG $\mathrm{E}_{\mathrm{EPI}, \mathrm{OSLD}}$ (as seen in Tab. 2), ob- tained by determining $\mathrm{COF}_{\mathrm{w}, \mathrm{i}, \mathrm{j}}$ using " $\delta_{\mathrm{EPID}, \mathrm{w}}$ ", were found to be similar to DLG ${ }_{I C D}$ and DLG ${ }_{\text {OSLD }}$, respectively as given in Table 1 .

\section{D DLG EPID $_{\text {map }}$}

In this study, the central $7 \times 7 \mathrm{~cm}^{2}$ region corresponding to the central $180 \times 180$ pixels of the $2 \mathrm{D}$ $D_{\text {LFPI }}$ map was evaluated by generating profiles averaged over each adjacent leaf pair as shown in Figure 4.

The analysis of the DLG values across the three central adjacent leaf pairs showed (Fig. 5) that the $D_{L G}$ ICD and DLG ${ }_{\text {OSLD }}$ values ranged from $1.04 \mathrm{~mm}$ to $1.28 \mathrm{~mm}$ and $1.08 \mathrm{~mm}$ to $1.24 \mathrm{~mm}$ with corresponding mean values of $1.12 \pm 0.14 \mathrm{~mm}$ and $1.13 \pm 0.09 \mathrm{~mm}$, respectively.

The profiles obtained across the seven central adjacent leaf pairs of $2 \mathrm{D} \mathrm{DLG}_{\text {EPID }}$ map showed that the DLG varied from a minimum of $1.03 \mathrm{~mm}$ to a maximum of $1.20 \mathrm{~mm}$ with a mean value of $1.10 \pm 0.06 \mathrm{~mm}$. The analysis of the DLG $\mathrm{EPID}_{\mathrm{E}}$ profiles taken along each of the three central adjacent leaf pairs across the field also showed substantial variation in the mean DLG values of each leaf pair
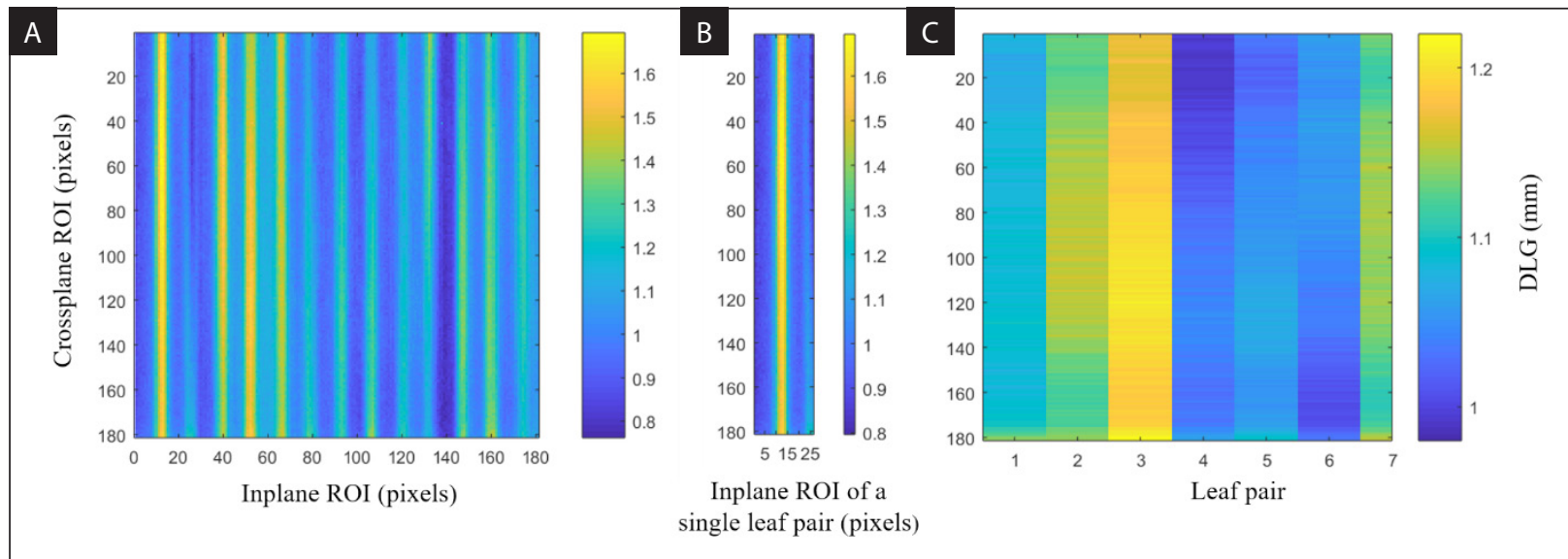

Figure 4. Analysis of the 2D DLG map. A. The central $7 \times 7 \mathrm{~cm}^{2}(180 \times 180$ pixels) region of the 2D DLG map selected for analysis; B. An adjacent leaf pair (width of 26 pixels) consisting of two adjoining MLC leaves (blue regions) that encompass an inter-leaf region in between (yellow region); C. The 2D map portraying the averaged leaf pair-specific DLG values across the field. The $3^{\text {rd }}, 4^{\text {th }}$ and $5^{\text {th }}$ leaf pairs correspond to the superior, central and inferior leaf pairs 


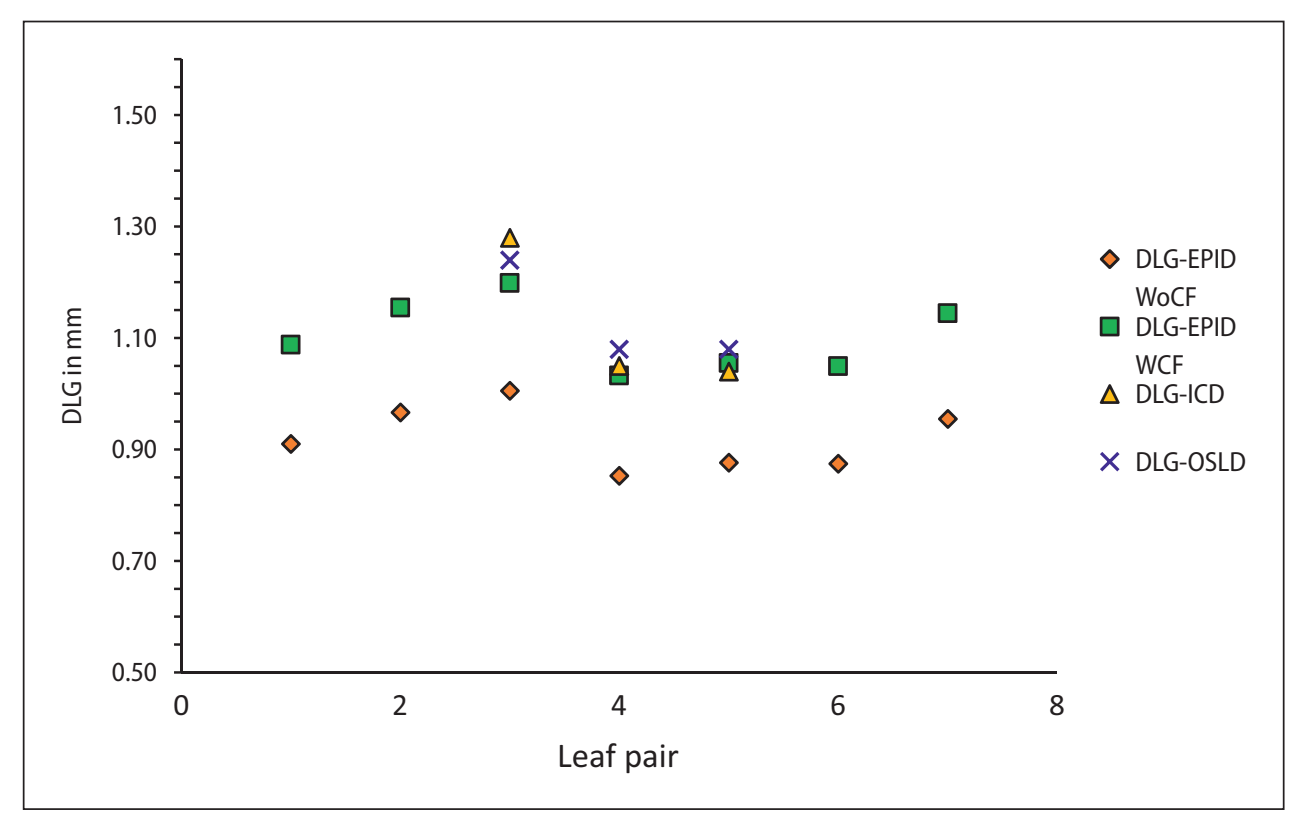

Figure 5. Leaf pair-specific dosimetric leaf gap (DLG) values obtained from the 2D DLG luminescence dosimeters (OSLD) and ionization chamber dosimetry (ICD); DLG-EPID WCF \& DLG-EPID WoCF — DLG and without correction factors. $3^{\text {rd }}, 4^{\text {th }} \& 5^{\text {th }} \mathrm{MLC}$ leaf pairs correspond to superior, central and inferior leaf pairs

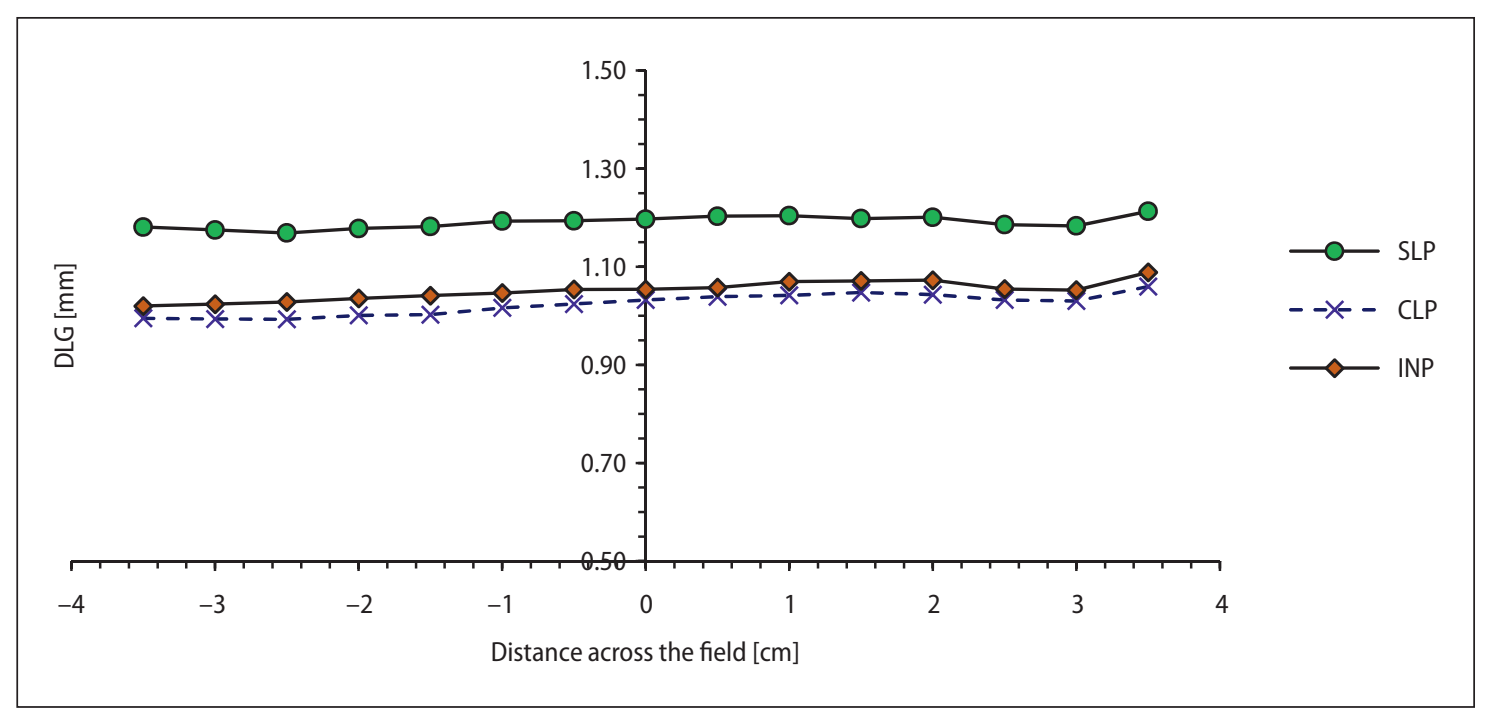

Figure 6. $D_{\text {LPID }}$ profile across the field for CAX and $\pm 1 \mathrm{~cm}$ OAX leaf pairs. SLP — superior leaf pair; CLP — central leaf pair; INP — inferior leaf pair

$(1.20 \pm 0.01 \mathrm{~mm}, 1.04 \pm 0.02 \mathrm{~mm}$ and $1.05 \pm 0.02$ $\mathrm{mm}$, respectively) and the same is demonstrated graphically in Figure 6.

\section{Discussion}

In common practice, the DLG measured at CAX is a unique value that is configured in the TPS during the beam data commissioning and applied glob- ally to all the leaf pairs during leaf motion calculation, while the DLG values at OAX have traditionally been ignored. However, in the present study, a new approach of using OSLD to measure the DLG at both the CAX and two OAX positions corresponding to six central MLC leaf pairs has been demonstrated with a simple dosimetric set-up. The conventional DLG $_{\text {ICD }}$, measured using the Semiflex ionization chamber was used to validate the DL- 
$\mathrm{G}_{\mathrm{OSLD}}$ and establish OSLD as its alternative, as well as determine the correction factors to improve the accuracy of the DLG $\mathrm{DPID}_{\mathrm{E}}$ values. Additionally, we have demonstrated a method to accurately compute the 2D DLG map from the portal images using custom-developed MATLAB scripts.

Even though the ICD and OSLD measurements were performed with diverse setups and in different media viz., water and PMMA, respectively, the results show similar variation in DLG values at each detector position for fixed measurement geometry, contributing to the knowledge of determining discrete DLG values for adjacent MLC leaf pairs. As the $\mathrm{DLG}_{\text {OsLD }}$ values seem to, by and large, mirror the reference DLG $_{\text {ICD }}$ values, the efficacy of OSLD with its high sensitivity in the low dose range, can be established.

It was also observed that the DLG derived using both the global and local normalization methods were in good agreement $(\leq 0.8 \%)$ with one another, irrespective of the type of detector used and the point of measurement. The local normalization was employed in the software script to compute the 2D $\mathrm{DLG}_{\mathrm{EPID}}$ map as it can be a more reliable method, especially in the OAX positions farther away from the CAX.

During the DLG $\mathrm{EPID}_{\mathrm{E}}$ measurements, the EPID displayed an under-response in the low dose ranges which resulted in the linear trend intercepting the horizontal dose axis at an earlier point in comparison with the corresponding ICD and OSLD measurements. This led to lower DLG $\mathrm{EPID}_{\mathrm{D}}$ values that were found to be consistent with the data reported previously [9]. Mei et al. suggested that the low dose insensitivity of the EPID could be due to the end MU effect of about $-0.4 \mathrm{MU}$ [12]. Another contributing factor for the low dose insensitivity could be the initial varying dose rate of the linear accelerator [28].

To further investigate the under-estimation of DLG $_{\text {EPID }}$, the sliding gap output factors measured using both EPID $\left(\mathrm{R}_{\mathrm{EPID}, \mathrm{w}}\right)$ and ICD $\left(\mathrm{R}_{\mathrm{ICD}, \mathrm{w}}\right)$ were analyzed. Interestingly, the relative dose response of EPID with respect to ICD was found to vary inversely as a function of " $w$ ". This led to the determination of " $\delta_{\text {EPID,W" }}$ (sliding aperture-specific correction) that accounted for the under-estimation of the DLG $\mathrm{DPID}_{\mathrm{EP}}$, thereby, establishing a harmony in results across different detectors, regardless of the position of measurement.
The 2D DLG $\mathrm{GPID}_{\text {EID }}$ map was computed for the central 20 adjacent leaf pairs using the portal images of the DLG plan comprising of static and sliding dynamic fields. The corrected output factor " $\mathrm{COF}_{\mathrm{w}, \mathrm{i}, \mathrm{j}}$ ", was calculated pixel-by-pixel, for the 7-layered 3D array that was generated by stacking the 7 portal images of the respective sweeping gap fields in the order of increasing gap width and the planar DLG map was generated using regression analysis. The 2D DLG $_{\text {EPID }}$ map has the additional advantage of determining the DLG value for each leaf pair at any position in the field.

In this study, only the central $7 \times 7 \mathrm{~cm}^{2}$ region out of the $10 \times 10 \mathrm{~cm}^{2}$ of the DLG map was analyzed (refer Fig. 2) as the penumbral regions could result in DLG values that are not meaningful [12]. It was observed that the DLG $\mathrm{BPID}_{\mathrm{E}}$ was different for each adjacent leaf pair in corroboration with the insight furnished by Kim et al., that the application of a solitary DLG value could prove to be insufficient due to the disparity between the radiological and physical leaf positions at various off-axis positions [15]. The maximum variation of the DLG $\mathrm{GPID}_{\mathrm{E}}$ across the 7 leaf pairs analyzed in our study was found to be $0.17 \mathrm{~mm}$ and this becomes important in the light of the conclusion by Luo et al., who reported that even an average positional error of $0.2 \mathrm{~mm}$ of the MLC could lead to a deviation of $1.0 \%$ of dose to the target. Hence, the varying DLG patterns across and along each leaf pair highlight the need for leaf pair-specific DLG values that must be incorporated into the TPS for more accurate calculation of fluence-modulated dose distributions. Kumaraswamy et al., who measured the $2 \mathrm{D}$ variation of the DLG using a diode matrix array detector [25], also evaluated and reported that the retrospective incorporation of the same into highly modulated VMAT plans helped in improving the gamma pass rate, especially in the outer regions with larger leaf width of $1 \mathrm{~cm}$ [26]. Although it was observed from the standard deviation that the variation of the DLG parallel to the direction of MLC motion was lesser when compared to variation of the DLG in the direction perpendicular to MLC motion, the need for determining and modelling OAX DLG values in the TPS should not be undermined.

Sophisticated treatment techniques such as IMRT and VMAT rely on accurate dose measurement and beam configuration of the calculation algorithm that includes the modelling of the dose 
profile width transmitted through the MLC leaf ends as the DLG parameter [29]. Driven by the fact that the measurement of the DLG can be tedious, the pursuit of a robust dosimetric system that can overcome existing limitations and accurately determine the DLG continues with great vigour.

In this study, the proximity of the mean DLG values obtained using the three different detectors shows that the OSLD, and EPID corrected for low-dose under-response, are comparable to ICD, proving to be suitable alternatives. Moreover, unlike for ICD, where the ten DLG fields must be delivered at each measurement location, the OSLD and EPID require the same to be delivered only once. While the OSLD can be used to determine the DLG accurately without an elaborate setup, the high resolution EPID with its quick setup does not require a read-out procedure to extract the dose information. Additionally, the EPID permits a greater degree of insight into the varying patterns of the DLG with respect to each leaf pair across the field. In future, the DLG plans can be created for the entire leaf bank to help determine the complete picture of OAX DLG, especially for the outer MLCs of larger widths that could encounter substantial variations.

\section{Conclusions}

The commensurate results of $\mathrm{DLG}_{\text {OSLD }}$ values with DLG $_{\text {ICD }}$ values at both CAX and OAX positions have proven the efficacy of OSLD as an appropriate dosimeter for DLG measurement. The ability to capture the low doses resulting from small sliding aperture gaps with a simple dosimetric set up and calibration promises a potential improvement in DLG measurement and verification. The under-estimation of DLG values due to the inherent limitation of EPID in the low dose ranges has been successfully addressed by the novel perception of using appropriate aperture-specific correction factors. The 2D DLG $\mathrm{EPID}_{\mathrm{D}}$ map highlights the potential importance of leaf-specific DLG values in order to accurately model the radiation transmission through the abutting leaves and help to stave off the common practice of employing a single DLG value to the entire leaf bank for commissioning of a modern TPS in future.

\section{Acknowledgements}

The authors are grateful to Dr Retna Ponmalar for her help in proof reading the manuscript.

\section{Conflict of interest}

None declared.

\section{Funding}

This work was supported by an internal Fluid Research Grant (FG/9307/2/2015) from Christian Medical College, Vellore.

\section{References}

1. Boyer AL, Biggs PJ, Galvin J, Klein EE, LoSasso T, Low D. Basic applications of multileaf collimators. American Association of Physicists in Medicine, Madison 2001.

2. Orlandini LC, Betti M, Fulcheri C, et al. Dosimetric impact of different multileaf collimators on prostate intensity modulated treatment planning. Rep Pract Oncol Radiother. 2015; 20(5): 358-364, doi: 10.1016/j.rpor.2015.06.005, indexed in Pubmed: 26549993.

3. Otto K. Volumetric modulated arc therapy: IMRT in a single gantry arc. Med Phys. 2008; 35(1): 310-317, doi: 10.1118/1.2818738, indexed in Pubmed: 18293586.

4. Arnfield MR, Siebers JV, Kim JO, et al. A method for determining multileaf collimator transmission and scatter for dynamic intensity modulated radiotherapy. Med Phys. 2000; 27(10): 2231-2241, doi: 10.1118/1.1312190, indexed in Pubmed: 11099190.

5. The Modern Technology of Radiation Oncology. Volume 3. In: Van Dyke J. ed. A Compendium for Medical Physicists and Radiation Oncologists. 1st ed. Medical Physics Publishing Corporation, Madison 2013.

6. Szpala S, Cao F, Kohli K. On using the dosimetric leaf gap to model the rounded leaf ends in VMAT/RapidArc plans. J Appl Clin Med Phys. 2014; 15(2): 4484, doi: 10.1120/ jacmp.v15i2.4484, indexed in Pubmed: 24710433.

7. Eclipse Algorithms_Reference Guide. Varian Medical Systems Inc, Palo Alto 2011.

8. Shende R, Patel G. Validation of Dosimetric Leaf Gap (DLG) prior to its implementation in Treatment Planning System (TPS): TrueBeam ${ }^{\text {TM }}$ millennium 120 leaf MLC. Rep Pract Oncol Radiother. 2017; 22(6): 485-494, doi: 10.1016/j. rpor.2017.09.001, indexed in Pubmed: 29070960.

9. Balasingh ST, Singh IR, Rafic KM, et al. Determination of dosimetric leaf gap using amorphous silicon electronic portal imaging device and its influence on intensity modulated radiotherapy dose delivery. J Med Phys. 2015; 40(3): 129-135, doi: 10.4103/0971-6203.165072, indexed in Pubmed: 26500398.

10. Esch AV, Bohsung J, Sorvari $P$, et al. Acceptance tests and quality control (QC) procedures for the clinical implementation of intensity modulated radiotherapy (IMRT) using inverse planning and the sliding window technique: experience from five radiotherapy departments. Radiother Oncol. 2002; 65(1): 53-70, doi: 10.1016/ s0167-8140(02)00174-3, indexed in Pubmed: 12413675. 
11. Clark CH. IMRT clinical implementation: Prostate and pelvic node irradiation using Helios and a 120-leaf multileaf collimator. J Appl Clin Med Phys. 2002; 3(4): 273, doi: 10.1120/1.1499095, indexed in Pubmed: 12383047.

12. Mei X, Nygren I, Villarreal-Barajas JE. On the use of the MLC dosimetric leaf gap as a quality control tool for accurate dynamic IMRT delivery. Med Phys. 2011; 38(4): 2246-2255, doi: 10.1118/1.3567148, indexed in Pubmed: 21626959.

13. LoSasso TJ. IMRT Delivery System QA. https://www.aapm. org/meetings/03SS/Presentations/Losasso.pdf.

14. Lin CY, Shiau AC, Ji JH, et al. A simple method for determining dosimetric leaf gap with cross-field dose width for rounded leaf-end multileaf collimator systems. Radiat Oncol. 2018; 13(1): 222, doi: 10.1186/s13014-018-1164-1, indexed in Pubmed: 30424789.

15. Kim J, Han JS, Hsia AnT, et al. Relationship between dosimetric leaf gap and dose calculation errors for high definition multi-leaf collimators in radiotherapy. Phys Imaging Radiat Oncol. 2018; 5: 31-36, doi: 10.1016/j. phro.2018.01.003, indexed in Pubmed: 33458366.

16. Amin MdN, Heaton R, Norrlinger $B$, et al. Small field electron beam dosimetry using MOSFET detector. J Appl Clin Med Phys. 2010; 12(1): 3267, doi: 10.1120/jacmp. v12i1.3267, indexed in Pubmed: 21330970.

17. Kumar A, Mukherjee G, Yadav G, et al. Optimized point dose measurement: An effective tool for QA in intensity-modulated radiotherapy. J Med Phys. 2007; 32(4): 156-160, doi: 10.4103/0971-6203.37480, indexed in Pubmed: 21224925.

18. Mesbahi A, Thwaites D, Reilly A. Experimental and Monte Carlo evaluation of Eclipse treatment planning system for lung dose calculations. Rep Pract Oncol Radiother. 2006; 11(3): 123-133, doi: 10.1016/s15071367(06)71057-4.

19. Bakhtiari M, Kumaraswamy L, Bailey DW, et al. Using an EPID for patient-specific VMAT quality assurance. Med Phys. 2011; 38(3): 1366-1373, doi: 10.1118/1.3552925, indexed in Pubmed: 21520847.

20. Jursinic PA. Characterization of optically stimulated luminescent dosimeters, OSLDs, for clinical dosimet- ric measurements. Med Phys. 2007; 34(12): 45944604, doi: 10.1118/1.2804555, indexed in Pubmed: 18196786

21. Smith L, Haque M, Morales J, et al. Radiation dose measurements of an on-board imager X-ray unit using optically-stimulated luminescence dosimeters. Australas Phys Eng Sci Med. 2015; 38(4): 665-669, doi: 10.1007/ s13246-015-0386-x, indexed in Pubmed: 26482528.

22. Ponmalar R, Manickam R, Ganesh KM, et al. Dosimetric characterization of optically stimulated luminescence dosimeter with therapeutic photon beams for use in clinical radiotherapy measurements. J Cancer Res Ther. 2017; 13(2): 304-312, doi: 10.4103/0973-1482.199432, indexed in Pubmed: 28643752.

23. Kairn T, Wilks R, Yu L, et al. In vivo monitoring of total skin electron dose using optically stimulated luminescence dosimeters. Rep Pract Oncol Radiother. 2020; 25(1): 35-40, doi: 10.1016/j.rpor.2019.12.011, indexed in Pubmed: 31889918.

24. Viamonte A, da Rosa LAR, Buckley LA, et al. Radiotherapy dosimetry using a commercial OSL system. Med Phys. 2008; 35(4): 1261-1266, doi: 10.1118/1.2841940, indexed in Pubmed: 18491518.

25. Kumaraswamy LK, Xu Z, Bailey DW, et al. Spatial variation of dosimetric leaf gap and its impact on dose delivery. Med Phys. 2014; 41(11): 111711-21, doi: 10.1118/1.4897572, indexed in Pubmed: 25370625.

26. Kumaraswamy LK, Xu Z, Bailey DW, et al. Evaluation of fluence-based dose delivery incorporating the spatial variation of dosimetric leaf gap (DLG). J Appl Clin Med Phys. 2016; 17(1): 12-21, doi: 10.1120/jacmp.v17i1.5883, indexed in Pubmed: 26894361.

27. LoSasso T, Chui CS, Ling CC. Physical and dosimetric aspects of a multileaf collimation system used in the dynamic mode for implementing intensity modulated radiotherapy. Med Phys. 1998; 25(10): 1919-1927, doi: 10.1118/1.598381, indexed in Pubmed: 9800699.

28. Winkler P, Hefner A, Georg D. Dose-response characteristics of an amorphous silicon EPID. Med Phys. 2005; 32(10): 3095-3105, doi: 10.1118/1.2040711, indexed in Pubmed: 16279061.

29. Xue J, Wang H, Barbee D, et al. A Practical Method to Optimize Quality Assurance Results of Arc Therapy Plans in Beam Modeling. J Med Phys. 2018; 43(2): 106-111, doi: 10.4103/jmp.JMP_144_17, indexed in Pubmed: 29962688. 